\title{
Dynamics in Ultrathin Films: Particle Tracking Microrheology of Lang- muir Monolayers
}

\author{
Laura J. Bonales, Hernán Ritacco, José E.F. Rubio, Ramón G. Rubio*, F. Monroy and Francisco \\ Ortega
}

Departamento de Química Física I, Facultad de Química, Universidad Complutense, 28040-Madrid, Spain

\begin{abstract}
Particle tracking has been shown to be a powerful technique for measuring bulk and interfacial rheology of fluids. The Brownian motion of microparticles trapped at interfaces is very sensitive to the viscosity of the subphase, and to the contact angle of the particles. The Stokes-Einstein relation is fulfilled if the friction factor is properly taken into account. The diffusion coefficient of the latex microparticles spread on surfactant monolayers allows one to calculate the shear viscosity of the monolayer using Danov's theory. Good agreement was found with previous results for monolayers of pentadecanoic acid. The method has also been used to study monolayers of n-dodecanol. Moreover, the shear viscosity of a polymer monolayer has been calculated by particle tracking, and the results show good agreement with data obtained by canal viscosimetry. The temperature dependence of the shear viscosity shows the existence of a glass transition for monolayers of poly(4-hydroxystyrene).
\end{abstract}

\section{INTRODUCTION}

Langmuir monolayers of surfactants, polymers and micro- or nanoparticles spread at fluid-fluid interfaces are confined to quasi-two dimensions. The thickness of the monolayer is of the order of the size of a molecule in the case of simple surfactants (a few Amstrongs), of the radius of gyration in the case of polymers (a few $\mathrm{nm}$ ), or of the size of the particles. Even for relatively simple molecules such us the fatty acids, complex phase diagrams have been described, with semicrystalline, hexatic, and disordered phases existing at different temperatures, $\mathrm{T}$, and surface pressures, $\Pi[1-3]$ $\left(\Pi(\Gamma, T)=\gamma_{0}(\Gamma, T)-\gamma(\Gamma, T), \gamma\right.$ being the surface ten-sion with the monolayer, $\gamma_{0}$ the corresponding value for the bare interface, and $\Gamma$ the surface concentration). Even though the phase diagram of uncharged insoluble polymer monolayers do not usually reveal such a complexity [4,5], the existence of a liquid-to-glass transition has been reported in the literature for this kind of systems[6]. Moreover, phase transitions have been reported for rod-like polymers [7] and polyelectrolytes [8,9]. The transition from disordered to solid-like hexagonal phases, mediated by hexatic phases, has been described in detail for monolayers of monodisperse microparticles [10,11], while the fluid-to-glass transition has been found for monolayers of polydisperse microparticles [12].

The situation is somewhat more complicated when the surfactant or the polymer is, at least, partially soluble. In this case the thermodynamic state of the monolayer is coupled to the subphase through adsorption/desorption processes $[13,14]$. This may make the equilibrium state difficult to obtain within typical experimental times specially when solutions of synthetic polymers or proteins are studied [15-17]. The situation is even more complex for the case of microparticles were an extremely slow adsorption kinetics is coupled with a very high reduction in surface Gibbs energy when the particles are trapped at the surface, thus leading to a long-

*Address correspondence to this author at the Departamento de Química Física I, Facultad de Química, Universidad Complutense, 28040-Madrid, Spain; E-mail: rgrubio@quim.ucm.es lived metastable state when the particles are spread at the interface $[18,19]$.

The possibility to use thermodynamic [20], spectroscopic $[21,22]$, optical [23,24], and scattering $[25,26]$ techniques, as well as computer simulations $[27,28]$ has lead to a relatively complete knowledge of the equilibrium behaviour of Langmuir monolayers. However, it has been shown that the surface pressure may be insensitive to the existence of some structural or conformational transitions within the interfacial region $[29,30]$. Furthermore, in most of the industrial and biological processes, the interfaces are not in equilibrium conditions, but subject to external perturbations (e.g. stirring). In these conditions, the behaviour of the system is ruled by the gradients of temperature and concentration developed [31]. Moreover, under non-equilibrium conditions, the surface tension becomes a tensor, and depends on the rate of strain even in the simple case of pure two-dimensional deformations [32]. It is now well accepted that this dynamic character of fluid interfaces plays a key role in understanding the stability of thin films, foams, suspensions and emulsions $[16,33]$, as well as the mobility of bubbles and drops in viscous fluids [34], the correlation between the structure of the monolayers and the corresponding one of its LangmuirBlodgett films [35], and in the stability of the biological membranes [36], and lung surfactants [37].

It is well stablished that the dynamics of the simplest fluid-fluid interface can be described in terms of several dynamic modes [38] illustrated in (Fig. 1). One type of motion is perpendicular to the interface plane, the capillary mode, and is governed by the gravity and the surface tension. Two more modes exist in the plane of the interface: a shear mode, and a compression (or dilatational) one.

Each of these two in-plane motions are characterized by a frequency dependent elasticity modulus $\varepsilon *(\omega)=\varepsilon_{\mathrm{R}}(\omega)+\mathrm{i} \varepsilon_{\mathrm{I}}(\omega)$, where the first term is the real part or elasticity, and the second term is the imaginary or loss 
modulus, that is given by the product of the frequency $\omega$ and the viscosity.

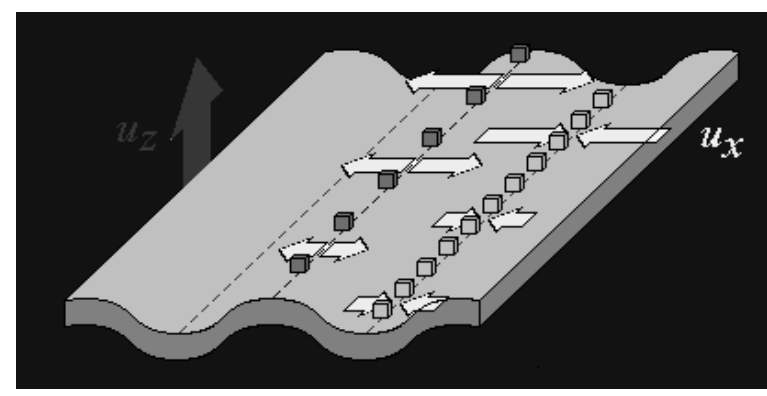

Fig. (1). Capillary (out of the interface plane) and dilatational(inplane) modes in a fluid-fluid interface. The cubes represent surfactant molecules.

Time-resolved versions of the scattering techniques, i.e. $\mathrm{x}$-ray photon correlation spectroscopy (XPCS) and neutron spin-echo (NSE), have emerged as powerful probes of the microscopic dynamics in these soft and fragile systems [39]. However, long-time relaxation phenomena, like collective diffusion, or more sophisticated mechanisms of relaxation like reptation motions, arm retractions, etc. show in some cases too slow dynamics to accomplish their coherence requirements. As a recent example, XPCS has been successfully used to follow slow collective dynamics in solidsupported thin polymer films close to the glass transition [40], however high-brilliance synchrotron sources are needed to carry out these experiments. Surface rheology is one of the best options to study the dynamics of slow processes at fluid interfaces. The experimental advances in the last decades allow the researcher to explore a rather broad frequency range: $1 \mathrm{mHz}-1 \mathrm{MHz}$ by combining different experimental techniques. Moreover, in the last two decades it has become possible to routinely study both the dilational and the shear rheology using commercial techniques, at least for $\omega<100$ $\mathrm{Hz}[29,38,41,42]$. In the case of dilational rheology, some of the techniques are affected by limitations due to the hydrodynamic corrections to be made to the raw data [43], or to incomplete modelling of the hydrodynamics of the interface, that sometimes lead to physically unsound results [44]. Also the techniques described for measuring the shear viscosity lead in some cases to inconsistent results for some systems. It must also be stressed that both in the case of dilational and shear rheometers, the experimental techniques are suitable only for limited ranges of frequencies or viscosities [45].
In recent years monitoring the trajectory of microparticles with video-microscopy techniques, and their analysis in terms of the basic equations of Brownian motion has opened the field so-called microrheology that has been applied both to three- [46] and two-dimensional systems [47]. The possibility of tuning the chemical nature of the microparticle's surface makes it possible to reduce or even eliminate the "stick-slip" problems that can be found in some concentrate monolayers when oscillatory shear rheometers are used. However, few applications to the rheology of monolayers have appear so far in the literature [48-53], and in general they deal with diffusion coefficients but do not calculate the surface shear viscosity, a non trivial problem as demonstrated by Danov et al. [54].

The purpose of this work is to explore the possibilities of the particle-tracking technique to measure the shear viscosity of Langmuir monolayers. To this end, the diffusion coefficient of latex microparticles will be measured in monolayers of insoluble surfactants (pentadecanoic acid, and 1dodecanol) and of polymers poly(4-hydroxystyrene). The data will be analyzed in terms of Danov's theory in order to obtain the shear viscosity, and the results will be compared with those obtained by other techniques, and with the dilational viscosity data.

\section{MATERIALS AND METHODOLOGY}

\section{Chemicals}

Poly(methyl methacrylate) microparticles were purchased from Interfacial Dynamics Their diameters were determined by dynamic light scattering (DLS, ALV-9000, Germany) and by scanning electronic microscopy (SEM, JEOL JSM 6335F). Particles with diameters ranging from 1 to $5 \mu \mathrm{m}$ have been used. The outer surface of the particles contains sulfate groups that are partially ionized in water suspensions. Table 1 shows the characteristics of the particles used.

Pentadecanoic acid (PDA), methanol, isopropanol, and ndodecanol were purchased from Aldrich. The water used was Milli-Q (Millipore) with a minimum resistence of $18 \mathrm{M} \Omega$. The microparticles were spreaded using a mixture of water and methanol $(1: 1 \mathrm{v} / \mathrm{v})$ as spreading solvent. In the case of the PDA and dodecanol monolayers, the spreading solvent was chloroform, while in the P4HS the solvent was a mixture of tetrahydrofurane and benzene $(5: 1 \mathrm{v} / \mathrm{v})$.

Table 1. Characteristics of the Microparticles Used in this Work

\begin{tabular}{|c|c|c|c|c|}
\hline & Mean diameter* $(\mu \mathrm{m})$ & $\begin{array}{c}\text { Standard deviation of diameter } \\
(\mu \mathrm{m})\end{array}$ & Charge content $* *(\mu \mathrm{Eq} / \mathrm{g})$ & $\begin{array}{c}\text { Surface charge density } \\
\left(\mu \mathrm{C} / \mathrm{cm}^{2}\right)\end{array}$ \\
\hline P1 & 1.0 & 0.042 & 3.4 & 5.8 \\
\hline P2 & 1.6 & 0.039 & 2.2 & 5.9 \\
\hline P3 & 2.9 & 0.13 & 2.0 & 9.7 \\
\hline P5 & 5.7 & 0.54 & 0.6 & 6.1 \\
\hline
\end{tabular}

*Determined from Transmission electron Microscopy.

**Measured from conductometric titration. 

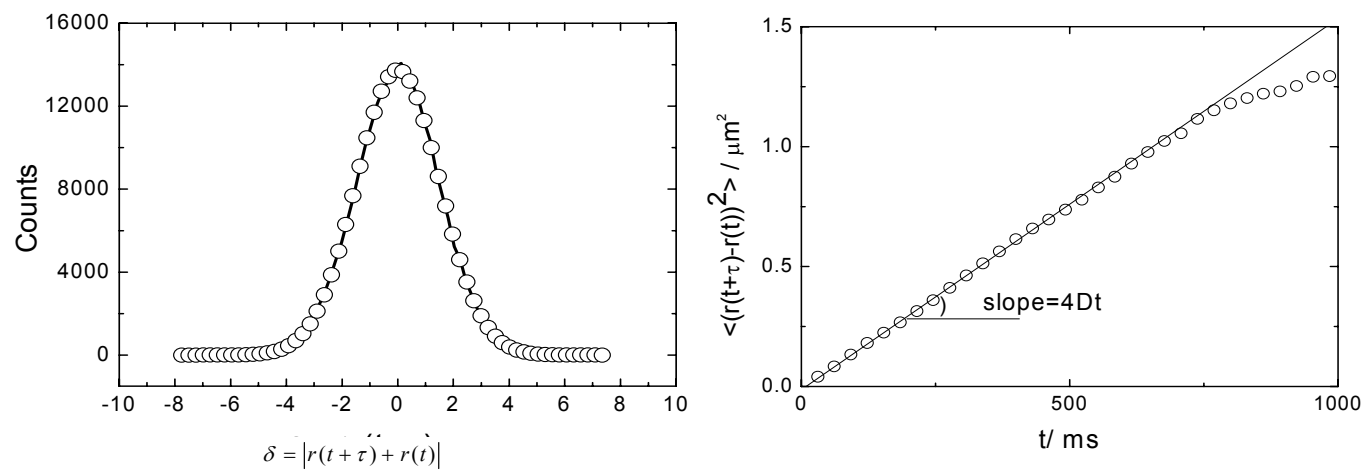

Fig. (2). Left: Gaussian shape of the displacements of a set of 250 particles for a time step $\tau=33 \mathrm{~ms}$. The line is the optimal fit to a Gaussian function. Right: Time dependence of the mean squared displacement of the particles. The slope of the initial part of the curve is $4 \mathrm{D}$ for a two dimensional system. The data correspond to $\tau=33 \mathrm{~ms}$.

\section{Particle Tracking Rheometer}

The equipment is based on a Nikon Eclipse 80-i microscope with a digital head (magnification from 0 to $2 \mathrm{x}$ ), and provided with 10x, 50x and $100 \mathrm{x}$ long-distance objectives. A CCD camera (Hamamatsu, model C8800-21CU) capable to take 30 to 100 frames per second (fps) is used to record the Brownian trajectories of the particles. In the present studies a small number of particles ( 2 to 6 ) were followed simultaneously. Each snapshot is treated according to the protocol suggested by Crocker and Grier [55]. By analysing a sequence of pictures one can obtain the mean squared displacement (MSD) of these particles for all accesible time steps: the minimum time step was $\tau=1 / \mathrm{fps}$, and the maximum was $\tau=(\mathrm{NI}-1) / \mathrm{fps}$, NI being the total number of images taken. For the calculation of the MSD due to pure diffusion, the relative motion of all possible pairs of particles was utilized. This procedure eliminates the non-diffusive drift motions.

According to the theory of Brownian motion, for a given value of $\tau$, a histogram of the counts corresponding to each displacement from the origin is given by a Gaussian function. (Fig. 2) shows the results obtained for $2 \mu \mathrm{m}$ particles spread at the water/n-octane interface (the trajectories of 250 particles were followed). As expected, the results can be fitted very well to a Gaussian function. The EinsteinSmoluchowsky equation can be written as

$\operatorname{MSD}(t)=2 d D t+K_{0}$

where $\mathrm{d}$ is the dimension of the system, and $\mathrm{K}_{0}$ is an additive offset that arises in part from measurement errors. Eq.(1) allows to obtain the diffusion coefficient $D$ from a plot of MSD vs. t (Fig. 2). In order to improve the precision of the data, each value of $\mathrm{D}$ represents the average of ten measurements at different positions of the interface. As it can be observed in (Fig. 2), the linear behaviour predicted by Eq.(1) holds only for the short time range. The deviations observed at higher t's arise from the poor statistics: the first point corresponds to the averaging of (NI-1) data points (the minimum time step available corresponds to one frame). When we increase the time step to $\mathrm{n}$ frames the average is reduced to (NI-n) data points, then the point for the highest $\mathrm{t}$ (where $\mathrm{n}$ = NI-1) corresponds to just a single datum; in any case, following the trajectories for larger $\tau$ 's allows one to extend the linear $t$ interval in the MSD vs. $t$ plot [49].

\section{Contact Angle Measurements}

The contact angle of particles at the air/water interface has been measured with the method described by Paunov et $a l$. [56]. The method takes advantage of the fact that increasing $\mathrm{T}$ induces a sol-gel transition in concentrated solutions of agarose. Agarose does not adsorb at the water/air surface, thus the surface tension of agarose solutions remains equal to that of pure water even at high concentrations. Therefore, one may expect that the value of $\theta$ for microparticles will be the same in water than in agarose solutions. The recipe is as follows: a) micro particles are spread on the surface of a concentrated agarose solution; b) the temperature is raised until gellification thus freezing the position of the particles at the interface; c) dimethylsiloxane is gently poured over the gel; d) the siloxane is cured to form a poly(dimethylsiloxane) (PDMS) phase over the agarose; e) the PDMS is peeled off taking the micro particles with it; f) a Scanning electronic microscopy (SEM) image of the particles on the PDMS is taken, which allows one to measure the contact angle. (Fig. 3) shows two typical images obtained using the method described above. This method allows one also to obtain an independent measure of the diameter of the particles complementary to that obtained by DLS. It can be observed that $\theta$ strongly depends on the characteristics of the particles (Table 1), and on the nature of the interface. This is very important because the value of $\theta$ has a dramatic effect on the shear viscosity obtained by the particle tracking technique (see below).

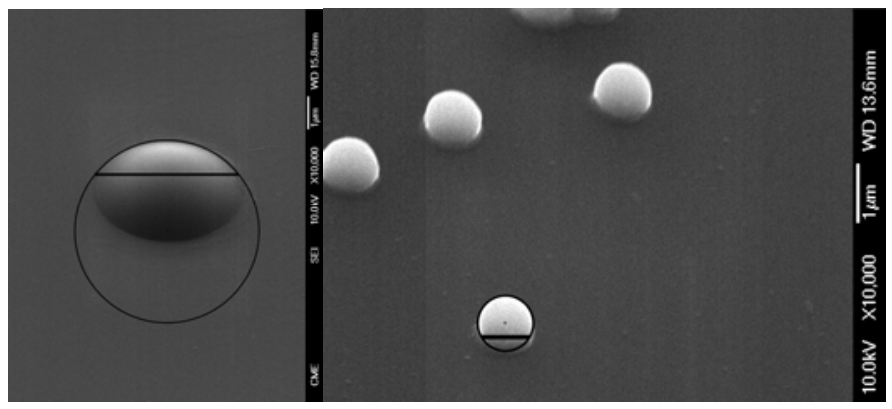

Fig. (3). SEM images of the two types of particles embedded in the siloxane polymer. Left panel:P1 particles at the water/air interface $\left(\theta=63 \pm 6^{\circ}\right)$. Right panel: $\mathrm{P} 4$ particles at the octane/water interface $(\theta=131 \pm 9)$. 


\section{RESULTS}

\section{The Air-Water Interface}

In order to test first the sensitivity of the technique to the bulk viscosity, we have studied the diffusion coefficient of $1 \mu \mathrm{m}$ particles at the air/water interface as a function of the temperature. Table 2 collects the results obtained from an average of 7 experiments.

Table 2. Diffusion Coefficients of $1 \mu \mathrm{m}$ Particles on the Water/Air Interface

\begin{tabular}{|c|c|}
\hline $\mathbf{T} / \mathbf{K}$ & $\mathbf{1 0}^{\mathbf{1 3}} \cdot \mathbf{D} / \mathbf{m}^{2} \cdot \mathbf{s}^{-\mathbf{1}}$ \\
\hline \hline 293.15 & $6.4 \pm 3$ \\
\hline 295.15 & $6.3 \pm 2$ \\
\hline 298.15 & $7.3 \pm 0.8$ \\
\hline 299.15 & $8 \pm 1$ \\
\hline 303.15 & $9.7 \pm 0.1$ \\
\hline 308.15 & $10 \pm 1$ \\
\hline
\end{tabular}

The generalized Stokes-Einstein equation relates the radius of a particle with $\mathrm{D}$ and the viscosity of the fluid $\eta$

$\mathrm{D}=\frac{\mathrm{k}_{\mathrm{B}} \mathrm{T}}{\mathrm{f}_{\mathrm{r}}}=\frac{\mathrm{k}_{\mathrm{B}} \mathrm{T}}{\mathrm{f} \pi \eta \mathrm{R}}$

where $f_{r}$ is the friction coefficient, and $f$ is a coefficient that depends on the friction area (and therefore on the contact angle). The main problem in applying Eq.(2) to particles at interfaces is that $\mathrm{f}$ is a rather complex function of the contact angle. Following Danov's theory one find that $\mathrm{f}$ varies with the contact angle $\theta$ between $\mathrm{f}=3$ for $\theta=90^{\circ}$ and $\mathrm{f}=4.4$ for $\theta=20^{\circ}$ [53]. The value of $\theta$ obtained by Paunov's method for these particles was $\theta=60 \pm 9$. On the other hand, the temperature dependence of the water viscosity is given by

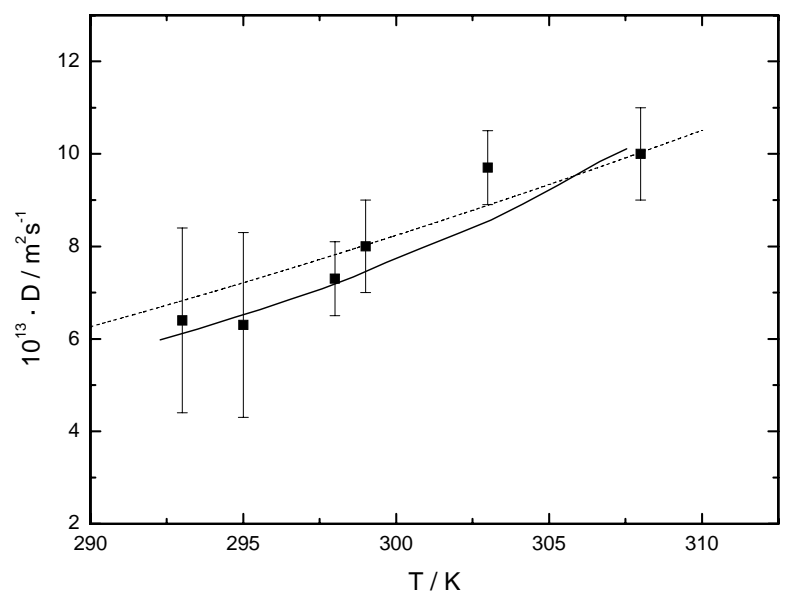

Fig. (4). Temperature dependence of the diffusion coefficients of 1 $\mu \mathrm{m}$ particles at the water/air interface. The symbols are the experimental results. The continuous curve is the prediction of Eqs.(2) and (3) with the contact angle measured by Paunov's method. The dashed line is the best fit to Eqs.(2) and (3) with a value of $f=3.76$.

$$
\left(\eta / \mathrm{g} \cdot \mathrm{cm}^{-1} \cdot \mathrm{s}^{-1}\right)=0.02767 \cdot 10^{-3} \exp \left[\frac{522.9}{(\mathrm{~T}-273.15)-298+150.64}\right]
$$

(Fig. 4) shows that there is a very satisfactory agreement between the experimental values of $\mathrm{D}$ and those calculated with Eqs. (2) and (3) using the experimental contact angle. Moreover, these results suggest that the present procedure may be a very convenient method for measuring the contact angle of microparticles at the air/water interface. In effect, using Eq.(3) and Eq.(2), and plotting D vs. T allows one to obtain the coefficient $f$. Using the value of $f$ in combination with Danov's theory [54] provides the value of $\theta$. For the results of (Fig. 4) one obtains $\mathrm{f}=3.76$ that corresponds to $\theta$ $=62^{\circ}$, which agrees within the experimental error with the value obtained using Paunov's method. Another way to look at the results is that combining experimental data for $\theta$ and Danov's theory makes particle tracking a rheometer sensitive enough for measuring the T-dependence of the bulk viscosity in small temperature ranges.

\section{Langmuir Monolayers of Surfactants}

The monolayers of pentadecanoic acid at the air/water interface have been extensively studied in the literature $[1,57]$. The $\Pi$ vs $1 / \Gamma$ isotherm at $22^{\circ} \mathrm{C}$ of (Fig. 5) shows that the monolayer presents a liquid-expanded (LE) to liquidcondensed (LC) phase transition (shown as a plateau at $\Pi \approx$ $6 \mathrm{mN} \cdot \mathrm{m}^{-1}$ ). (Fig. 6) shows the time dependence of the MSD for a value of $\Gamma$ corresponding to the LE phase close to the LE-LC transition. The two lines represent the tracking of the trajectories of individual particles, and of the relative motion of pairs of particles. The last method has the advantage that any global drift (due to air currents or to local heating) can be removed [50]. As it can be observed, the slope of both lines, and therefore the values of $\mathrm{D}$, is essentially the same.

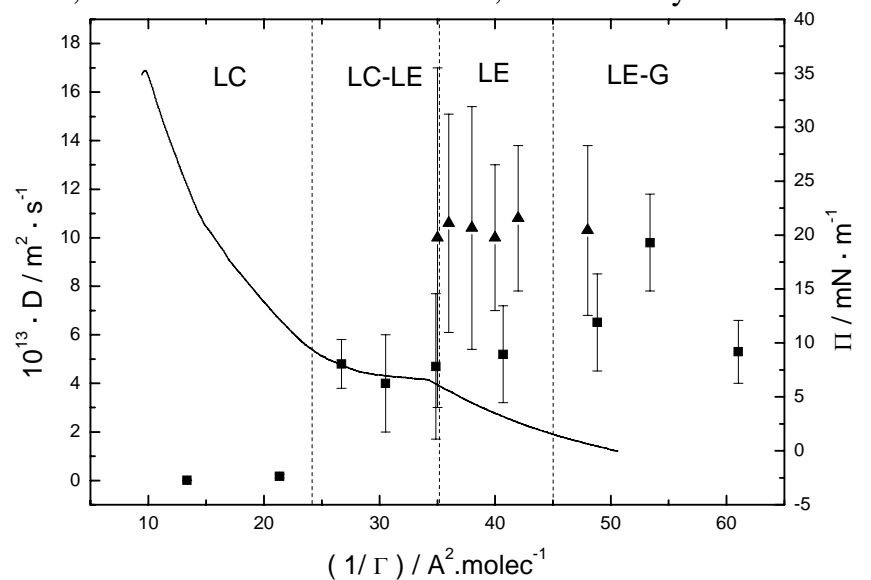

Fig. (5). Isotherm of the PDA monolayer at $22{ }^{\circ} \mathrm{C}$ (continuous line), and self-diffusion coefficients obtained from particle tracking of 1 $\mu \mathrm{m}$ particles (squares), and the values reported by Sickert and Rondelez for the same system [49] (triangles).

(Fig. 5) also includes the diffusion coefficient obtained from particle tracking with $1 \mu \mathrm{m}$ latex particles, as well as the values reported by Sickert and Rondelez for the dilute range of the same monolayer using polystyrene particles of 1 $\mu \mathrm{m}\left(\theta=50^{\circ}\right)$ [49]. The agreement between both sets of results is satisfactory within the combined experimental uncertainties. A significant result is that for the PDA monolayer D seems to be independent of $\Gamma$ in the LE and the LE-LC re- 
gion within the experimental uncertainty. As expected, D decreases significantly in the $\mathrm{LC}$ region.

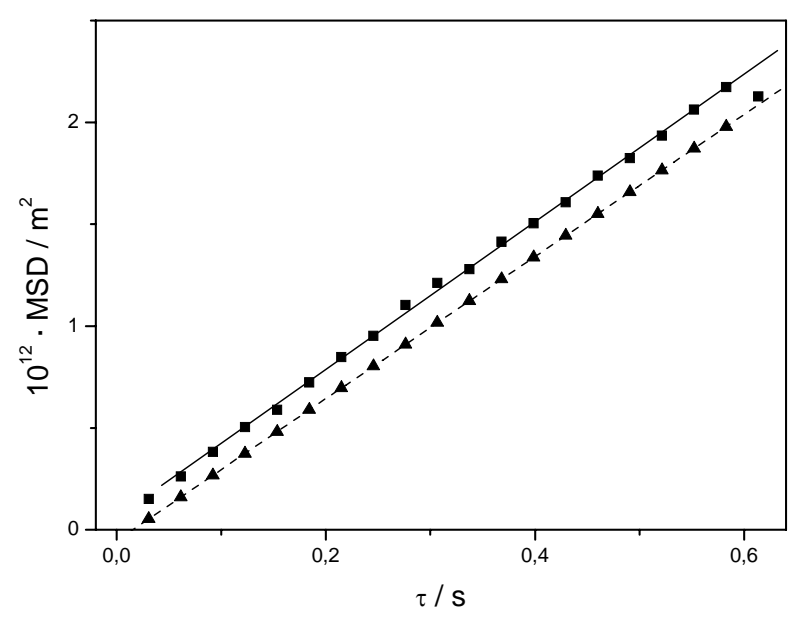

Fig. (6). Time dependence of the MSD of $1 \mu \mathrm{m}$ particles on a PDA monolayer for a value of $\Gamma$ within the LE region close to the LE-LC transition at $22{ }^{\circ} \mathrm{C}$. The squares correspond to the tracking of the trajectories of single particles, while the circles to the relative trajectories of pairs of particles.

(Fig. 7) shows the relationship between D and the surface shear viscosity $\eta_{\mathrm{s}}$ for different values of the contact angle according to Danov's theory [54].

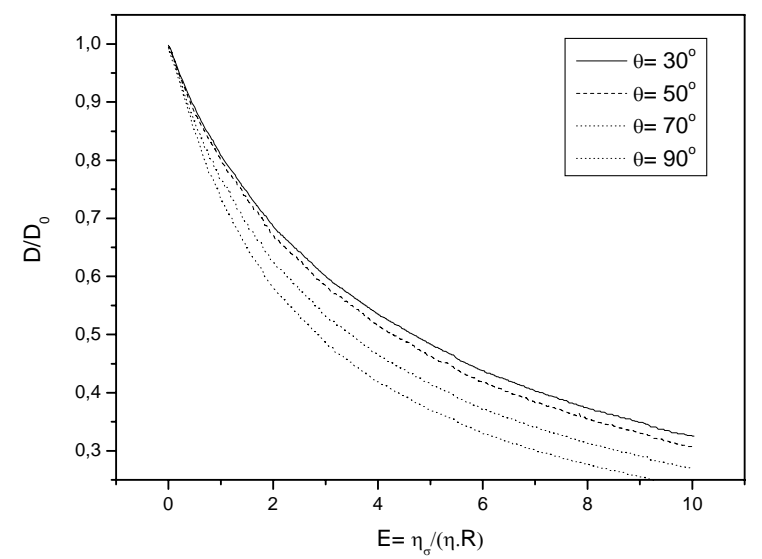

Fig. (7). Surface shear viscosity dependence of the diffusion coefficient of particles according to Danov's theory [54] for different values of the contact angle. $\mathrm{D}_{0}$ is the diffusion coefficient of the particles on the bare water/air surface, $\eta$ is the bulk viscosity of the subphase, and $\mathrm{R}$ is the radius of the particle.

Using the experimental value of the contact angle, and the values of $\mathrm{D} / \mathrm{D}_{0}$, (Fig. 7) allows one to calculate the values of $\eta_{\mathrm{s}}$ plotted in (Fig. 8). As expected from the results of D, $\eta_{\mathrm{s}}$ remains almost constant in the LE and the LE-LC regions, and increases abruptly almost three orders of magnitude in the $\mathrm{LC}$ region. As pointed out by Sickert and Rondelez, the constancy of $\eta_{\mathrm{s}}$ in the LE and LE-LC regions is unexpected, and it is most probably due to the existence of two antagonistic effects: the increase of density, that would decrease D and increase $\eta_{\mathrm{s}}$, and the ordering of the hydrocarbon chains that increase their length and decrease their tilt angle with respect to the interface [58].

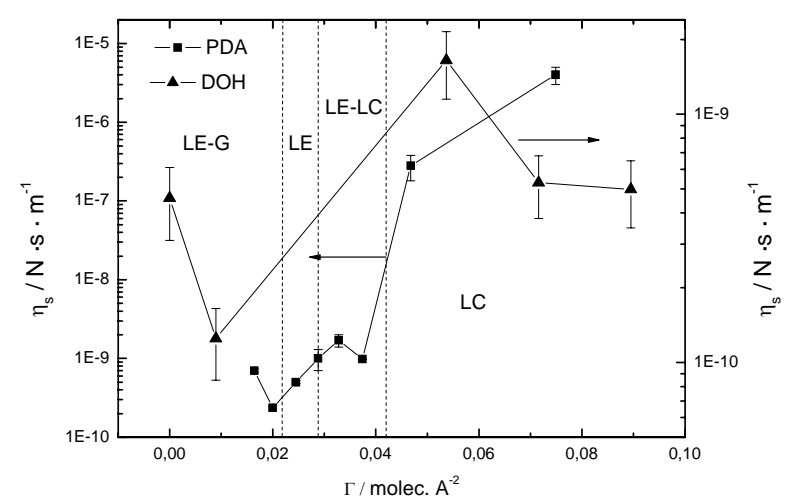

Fig. (8). Shear viscosity for surfactant monolayers of PDA and $\mathrm{DOH}$ at the air/water interface obtained from the diffusion coefficients of $1 \mu \mathrm{m}$ latex microparticles. The vertical dashed lines indicate the limits between the different regimes of the PDA monolayer.

It is well known that long-chain $\mathrm{n}$-alcohols for $\mathrm{m}$ stable Langmuir monolayers at the air/water surface, with a complex 2-D phase diagram qualitatively similar to that of the fatty acids and esters [59]. Moreover, at the spreading pressure, surface induced solidification has been reported for some of the monolayers $[60,61]$. We have studied the monolayer of $\mathrm{n}$-dodecanol at $22{ }^{\circ} \mathrm{C}$ over the whole $\Gamma$ range. $\mathrm{n}$ Dodecanol is at the border between soluble and insoluble monolayers, that makes the distinction between Langmuir and Gibbs monolayers somewhat fuzzy. The isotherm shown in (Fig. 9) points out the existence of a surface phase transition similar to that previously discussed for the PDA monolayer. D apparently increases as the area is decreased, and then there is a strong decrease after the surface phase transition. However, the error bars of the diffusion coefficients in the dilute region are quite large, and therefore no further discussion can be done. The values of $\eta_{\mathrm{s}}$ are plotted in (Fig. 8), showing the same qualitative behaviour than the monolayer of PDA.

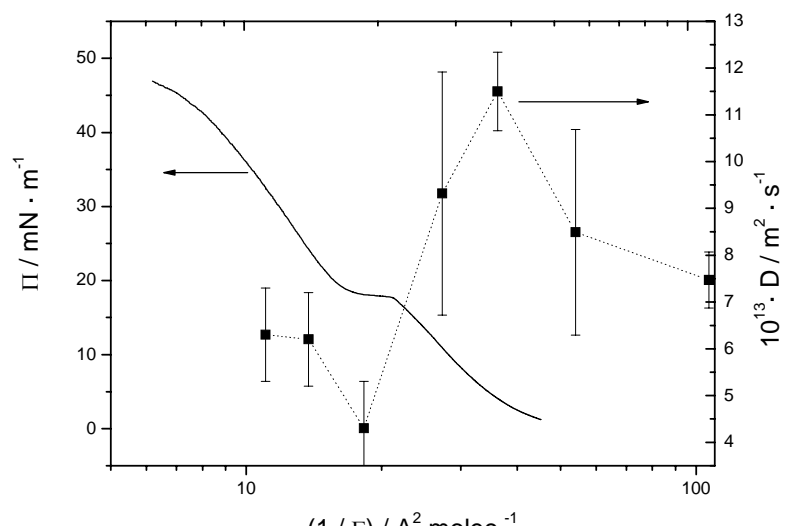

$(1 / \Gamma) / A^{2} \cdot$ molec $^{-1}$

Fig. (9). Isotherm and diffusion coefficient of $1 \mu \mathrm{m}$ particles on a ndodecanol monolayer at $22{ }^{\circ} \mathrm{C}$.

In a recent work, Liggieri et al. [62] have studied the dilational complex elasticity of Gibbs monolayers of $n$ dodecanol at $10{ }^{\circ} \mathrm{C}$. They found that the elasticity can be explained if one takes into account that there is an aggregation process responsable for the surface phase transition. As expected, the elasticity modulus presents a minimum at the 

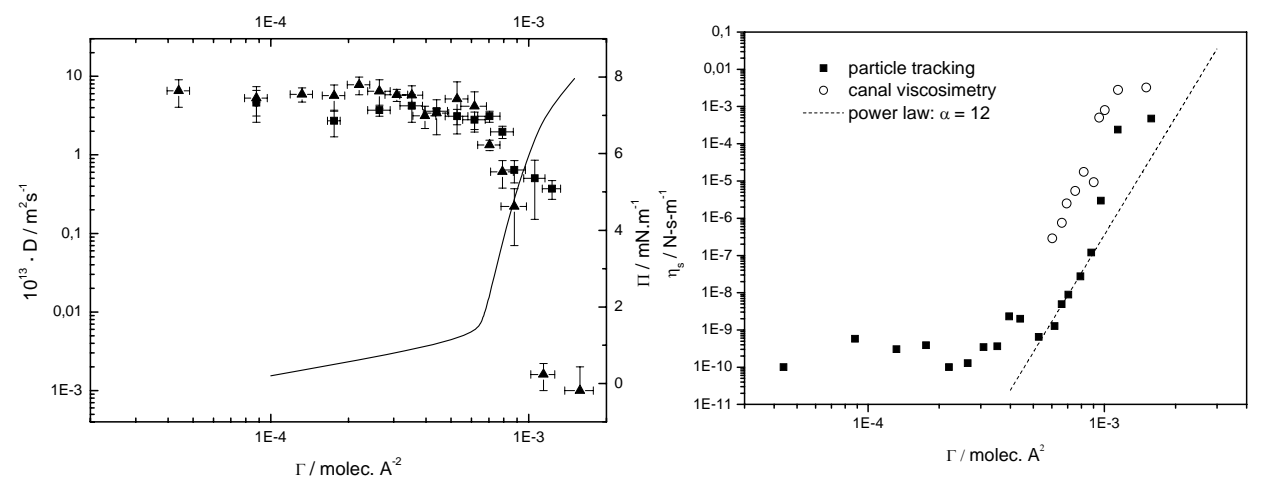

Fig. (10). Monolayers of P4HS onto water. Left: Diffusion coefficients obtained by the particle tracking method ( triangles $2.9 \mu \mathrm{m}$ particles, squares $1 \mu \mathrm{m}$ particles) and the $\Pi$ vs. $\Gamma$ isotherm at $22{ }^{\circ} \mathrm{C}$ (continuous line). Right: Shear viscosity obtained by particle tracking (full squares), and by canal viscosimetry (open circles) as a function of the surface concentration. The power law behaviour in the semidilute regime is shown by the dashed line.

phase transition, and then increases with the surface pressure.

\section{Langmuir Monolayers of Insoluble Polymers}

Polymer monolayers are important for building thin films by the Langmuir-Blodgett [63] or by electrostatic selfassembling methods [64]. The study of the dilational viscoelastic moduli of Langmuir polymer monolayers has pointed out the existence of a complex spectrum of relaxation modes [65]. In a broad frequency range, the dilational viscosity shows a strong frequency dependence, that can be roughly described by the following power law $\kappa \sim \omega^{-1}[45,65]$. Using relaxation experiments, Hilles et al. [66] have shown that polymer monolayers undergo a fluid to glass transition (GT) at a well defined temperature when the $\Gamma$ is in the so-called semidilute regime [66]. It is well known that in 3D systems the shear viscosity dramatically increases near the GT [67]. We have studied $\eta_{\mathrm{s}}$ for the monolayers of the same polymer studied in Ref. [66], poly(4-hydroxy styrene) (P4HS), using both particle tracking and canal viscosimetry. (Fig. 10) shows the surface concentration dependence of the diffusion coefficient of $1 \mu \mathrm{m}$ and $2.9 \mu \mathrm{m}$ particles at $22{ }^{\circ} \mathrm{C}$ and over the whole concentration range. Size differences lead to different particle velocities (and thus shear rates) and displacement magnitudes (thus strain amplitudes). The fact that the $\mathrm{D} / \mathrm{D}_{0}$ results collapse on the same curve for the two particle sizes indicates that the tracking experiments are carried out within the linear viscoelastic regime of the monolayer. It can be observed that $\mathrm{D}$ decreases dramatically as $\Gamma$ increases, and the monolayer enters the semidilute regime. Such effect is expected as a synergetic combination of the density increase and the glass transition that takes place at $\mathrm{T}$ low enough as a result of the percolation of the system [66].

The use of Danov's theory allows one to calculate $\eta_{\mathrm{s}}$, although it must be taken into account that most of the surface concentrations lead to values of $\mathrm{D} / \mathrm{D}_{0}$ below 0.2 , thus corresponding to small slopes in the $D / D_{0}$ vs. $E=\frac{\eta_{s}}{\eta \cdot R}$ curves (Fig. 7), which increases the uncertainty in the determination of $\eta_{\mathrm{s}}$. Fig. (10) shows that the values of $\eta_{\mathrm{s}}$ increase by seven orders of magnitude in the semidilute and concentrated regimes. The exponent of the power law followed in the semidilute rante is $\alpha=12$, that corresponds to a polymer in rather poor solvent conditions, in agreement with the conclusions previously obtained from the analysis of the $\Pi-\Gamma$ isotherm and of the dilational viscoelastic moduli [45].

We have also used a canal-type viscometer [68] to obtain $\eta_{\mathrm{s}}$. Fig. (10) shows that the results compare favourably with those obtained by particle tracking, taking into account that there is a three degrees temperature difference between both sets of data (see below).

In a recent work we showed that the P4HS monolayer undergoes a fluid-to-glass transition as $\mathrm{T}$ was lowered [66]. Fig. (11) shows the T-dependence of the area occupied by the polymer at constant surface pressure. The sharp change of the slope is clearly indicative of the glass transition $[67,69]$, and it is similar to the plots obtained by Keddy et al. [70], and Forrest et al. [71] for thin polymer films from ellipsometric measurements. It must be stressed that the value of the glass transition temperature, $\mathrm{T}_{\mathrm{g}}$, obtained from (Fig. 12) (equilibrium measurements) is quite close to the one reported in Ref. [65] from relaxation experiments (rheological measurements). The value of $\mathrm{T}_{\mathrm{g}}$ for the Langmuir monolayer is much lower than the value reported for the bulk polymer $\left(\mathrm{T}_{\mathrm{g}} \sim 130^{\circ} \mathrm{C}\right)$ [72]. This result is also in agreement with the results of Forrest et al. for unsupported polymer thin films [71].

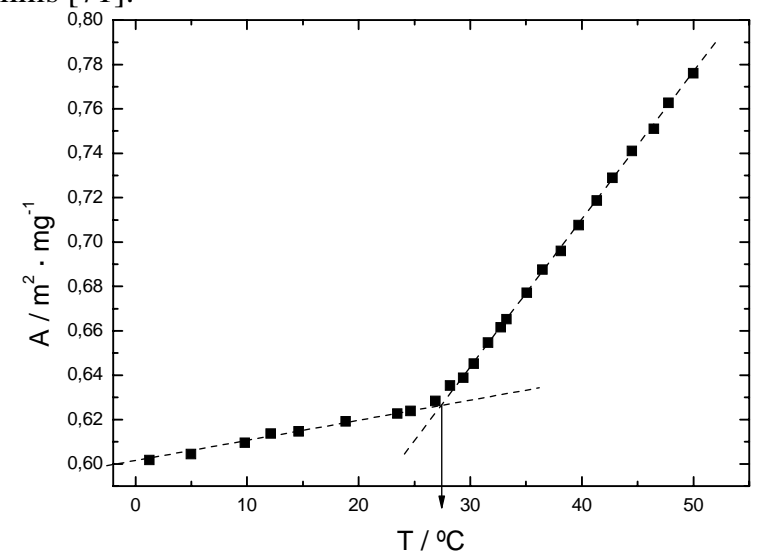

Fig. (11). Isobaric compression of a Langmuir film of P4HS at $\Pi=$ $6 \mathrm{mN} \cdot \mathrm{m}^{-1}$. The glass transition temperature is identified as the crossing point of the two straight lines. 
Fig. (12) shows the temperature dependence of $\eta_{\mathrm{s}}$ for two values of $\Gamma$ slightly above the overlapping concentration, $\Gamma^{*}$, i.e. the transition from the dilute to the concentrated regime, and for a value of $\Gamma$ in the dilute regime. The first point to remark is that for $\Gamma>\Gamma^{*}$ one finds a change in slope at $\mathrm{T} \sim$ $30^{\circ} \mathrm{C}$, i.e. the $\mathrm{T}_{\mathrm{g}}$ of the monolayer. The increase of the apparent expansivity near $\mathrm{T}_{\mathrm{g}}$ is a typical behaviour of bulk polymers [73]. However, for the dilute regime such transition is not observed for any $\mathrm{T}>0{ }^{\circ} \mathrm{C}$. The fact that the glass transition is triggered by decreasing $\mathrm{T}$ only for concentrations high enough is a well known fact in 3D colloidal suspensions [74]. Also a density dependence of $T_{g}$ for bulk polymers has been described in the literature by increasing the pressure [75], however in such experiments it is not possible to tune the density over a range as broad as in the Langmuir monolayers.

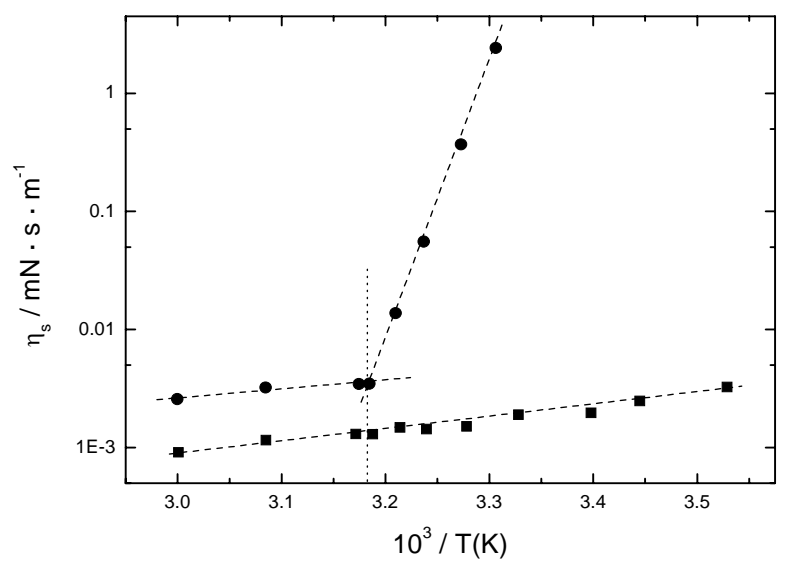

Fig. (12). Temperature dependence of the shear viscosity of a monolayer of P4HS. Circles correspond to $\Pi=8 \mathrm{mN} \cdot \mathrm{m}^{-1}$, i.e. above the overlapping concentration, and squares correspond to $\Pi=$ $2 \mathrm{mN} \cdot \mathrm{m}^{-1}$.

As already mentioned in the introduction, the in-plane dynamics of a fluid interface contains two different contributions: shear and dilational $[38,45,54]$. In the dydrodynamic modelling of the interface, necessary to analyze the surface wave experiments [38], it is usually assumed that the shear elasticity and viscosity are much smaller than the corresponding dilational ones [45,76,77]. Monroy et al. [45] and Rivillon et al. [65] have discussed the frequency, $\omega$, and composition dependence of the dilational viscosity, $\kappa$. In Ref. [45] they report $\kappa \sim 10^{-8}-10^{-7} \mathrm{mN} \cdot \mathrm{s} \cdot \mathrm{m}^{-1}$ for $\Gamma \leq 0.06$ molec $\cdot A^{-2}$. Assuming the validity of the $\kappa \sim \omega^{-1}$ law reported in Refs. [45] and [65], it would lead to $\kappa \sim 10^{-5}-10^{-4}$ $\mathrm{mN} \cdot \mathrm{s} \cdot \mathrm{m}^{-1}$ for $\omega=1 \mathrm{~Hz}$. This value is more than four orders of magnitude higher than $\eta_{\mathrm{s}}$ for $\Gamma<6 \cdot 10^{-4} \mathrm{molec} \cdot \mathrm{A}^{-2}$ (see Fig. 10); however $\kappa$ and $\eta_{\mathrm{s}}$ are similar for $\Gamma \geq 10^{-3} \mathrm{molec} \cdot \mathrm{A}^{-2}$, i.e. near the limit between the semidilute and the concentrated regimes of the monolayer. This fact may strongly complicate the analysis of the surface wave experiments in concentrated polymer monolayers. In fact, under these conditions it would be necessary to combine the surface wave experiments (that strictly speaking provide a sum of the shear and the dilational viscosity) with other that would provide either $\eta_{\mathrm{s}}$ or the dilational viscosity. It also may introduce some difficulties in the calculation of $\eta_{\mathrm{s}}$ from the $\mathrm{D} / \mathrm{D}_{0}$ data provided by the particle tracking technique [54]. A more

systematic comparison of $\kappa$ and $\eta_{\mathrm{s}}$ in a larger set of monolayers is required to clarify this point.

\section{CONCLUSIONS}

Particle tracking of latex microparticles at fluid interfaces has been found to be very sensitive to the viscosity of the subphase. The diffusion coefficient strongly depends on the contact angle that affects the friction coefficient.

Particles can be used as probes on surfactant and polymer monolayers. The Brownian motion of the microparticles allows one to calculate the shear viscosity of the monolayer by measuring the diffusion coefficient of the particles in combination with Danov's theory. The agreement found with the shear viscosity measured by canal viscosimetry is good. The results obtained for a monolayer of poly(4-hydroxystyrene) point out the existence of a glass transition at a temperature very close to that previously reported by relaxation experiments.

\section{ACKNOWLEDGMENTS}

This work was supported in part by MEC under grants CTQ2006-6208/BQU, FIS2006-12281-C02-01, and HA-20060019, and by CAM under grant INTERFASES S05-MAT227, CAM-UCM under grant 910591, and ESA under grant MAP 10-99-052. H. Ritacco was supported by MEC under a Juan de la Cierva contract, and L.J. Bonales under a FPI predoctoral grant. We thank H.M. Hilles for help with some of the experiments.

\section{REFERENCES}

[1]

[14] Kukhin, S.S.; Kretzschmar, G., Miller, R. Dynamics of adsorption at liquid interfaces; Elsevier: Amsterdam, 1995.

[15] Noskov, B.A.; Lin, S-Y., Loglio, G., Rubio, R.G., Miller, R. Langmuir, 2006, 22, 2647.

[16] Svitova, T.F., Radke, C.J. Ind. Eng. Chem. Res., 2005, 44, 1129.

[17] Ritacco, H., Cagna, A., Langevin, D. Colloids Surfaces A 2006, 282, 203.

[18] Miller, R., Feinerman, V.B., Kovalchuk, V.I., Grigoriev, D.O., Leser, M.E., Michel, M. Adv. Colloid Interf. Sci., 2006, 128-130, 17 ,

[19] Subramaniam, A.B.; Abkarian, M., Stone, H.A. Nat. Mat., 2005, 4, 553.

[20] Modern characterization methods of surfactant systems, Binks, B.P. Ed.; Marcel Dekker: New York, 1999.

[21] Gruniger, H., Möbius, D., Meyer, H. J. Chem. Phys., 1983, 79, 3701 .

[22] Mendelsohn, R., Brauner, J.W., Gericke, A. Аnnu. Rev. Phys. Chem,. 1995, 46, 305. 
[23] Tompkins, H.G.; A user's guide to ellipsometry; Academic Press: San Diego, 1993.

[24] Meunier, J. Colloids Surf. A 2000, 171, 33 .

[25] Tolan, M. X-Ray scattering from soft-matter thin films; SpringerVerlag: Berlin, 1999.

[26] Zhou, X.L.,Chen, S.H. Phys. Rep., 1995, 257, 223.

[27] Yethiraj, A. Macromolecules, 2003, 36, 5854

[28] Ostrovsky, B., Smith, M.A., Bar-Yam, Y. Int. J. Mod. Phys. C, 1997, 8, 93.

[29] Liggieri, L., Ferrari, M., Mondelli, D., Ravera, F. Faraday Discuss. 2005, 129, 125.

[30] Noskov, B.A.; Akentiev, A.V.,Bilibin, A.Yu., Zorin, I.M., Miller, R. Adv. Colloid Interf. Sci., 2003, 104, 245.

[31] Linde, H.; Velarde, M.G., Waldhelm, W., Loeschcke, K., Wierschem, A. Ind. Eng. Chem. Res., 2005, 44, 1396.

[32] Noskov, B.A., Loglio, G. Colloids Surf. A 1998, 143, 167.

[33] Bauget, F., Langevin, D., Lenormand, R. J. Colloid Interf. Sci. 2001, 239, 501.

[34] Koehler, S.A.; Hilgenfelt, S., Weeks, E.R., Stone, H.A. Phys. Rev. E. 2002, 66, 040601(R)

[35] Buhaenko, M.R., Richardson, R.M. Thin Solid Films, 1998, 159, 231.

[36] Boal, D. Mechanics of the cell; Cambridge Univ. Press.: Cambridge, 2002; Mathews, C.K.; van Holde, K.E., Ahern, K.G., Biochemistry, $3^{\text {rd }}$ Ed.; Benjamin/Cummings: N.Y., 2002.

[37] Wüstneck, R., Perez-Gil, J, Wüstneck, N, Cruz, A., Fainerman, V.N., Pison, U. Adv. Colloid Interf. Sci. 2005, 117, 33.

[38] Langevin, D. Ed., Light scattering by liquid surfaces and complementary techniques; Marcel Dekker: New York, 1992.

[39] Sikharulidze, I., Farago, B., Dolbnya, I.P., Madsen, A., de Jeu, W.H. Phys. Rev. Lett., 2003, 91, 65504.

[40] Kim, H., Ruhm, A., Lurio, L.B., Basu, J.K., Lal, J., Lumma, D., Mochrie, S.G.J., Sihna, S.K. Phys. Rev. Lett. 2003, 90, 068302.

[41] Monroy, F., Ortega, F., Rubio, R.G. J. Phys. Chem. B, 1999, 103, 2061.

[42] Gravranovic, G.T., Fuller, G.G. Faraday Discuss., 2005, 129, 1.

[43] Barentin, C., Ybert, Ch., di Meglio, J-M., Joanny, J-F. J. Fluid Mech. 1999, 397, 331.

[44] Monroy, F., Muñoz, M.G., Rubio, J.E.F., Ortega, F., Rubio, R.G. J. Phys. Chem. B 2002, 106, 5636.

[45] Monroy, F., Rivillon, S., Ortega, F., Rubio, R.G. J. Chem. Phys. 2001, 115, 530 .

[46] Waigh, T.A. Rep. Prog. Phys., 2005, 68, 685.

[47] Melle, S., Fuller, G.G., Rubio, M.A. Phys. Rev. E 2000, 61, 4111.

[48] Saxton, M.J.; Jacobson, K. Annu. Rev. Biophys. Biomol. Struct. 1997, 26, 373

Received: August 21, 2007

Revised: November 12,2007

Accepted: November 14, 2007
[49] Sickert, M., Rondelez, F. Phys. Rev. Lett., 2003, 90, 126104.

[50] Forstner, M.B.; Käs, J., Martin, D. Langmuir, 2001, 17, 567.

[51] Zahn, K., Méndez-Alcaraz, J.M., Maret, G. Phys. Rev. Lett., 1997, $79,175$.

[52] Montiel, D., Cang, H.,Yang, H. J. Phys. Chem. B, 2006, 110, 19763.

[53] Lin, B.; Rice, S.A., Weitz, D.A. Phys. Rev. E 1995, 51, 423

[54] Danov, K., Aust, R., Durst, F., Lange, U. J. Colloid Interf. Sci., 1995, 175,36

[55] Crocker, J.C., Grier, D.G. J. Colloid Interf. Sci., 1996, 179, 298.

[56] Paunov, V.N., Binks, B.P., Sabih, N.P. Langmuir, 2002, 18, 6946.

[57] Knobler, C.M. Adv. Chem. Phys., 1990, 77, 397.

[58] Alonso, C., Zasadzinski, J.A. J. Phys. Chem. B, 2006, 110, 22185.

[59] Vollhardt, D.; Feinerman, V.B., Emrich, G. J. Phys. Chem. B, 2000, 104, 8536

[60] Zakri, C., Renault, A., Rieu, J.P., Vallade, M., Berge, B., Legrand, J.F., Vignault, G., Grübel, G. Phys. Rev. B 1997, 55, 14163

[61] Muñoz, M.G., Luna, L., Monroy, F., Rubio, R.G., Ortega, F. Langmuir, 2000, 16, 6657.

[62] Liggieri, L., Ravera, F., Ferrari, M. Langmuir 2003, 19, 10233.

[63] Ulman, A., Ultrathin Organic Films; Academic Press: Boston, 1991.

[64] Multilayer thin films, Decher, G., Schlenoff J.B., Eds.; WileyVCH: Weinheim, 2003.

[65] Rivillon, S., Monroy, F., Ortega, F., Rubio, R.G. Eur. Phys. J. E 2002, 9,375

[66] Hilles, H.M., Ortega, F., Rubio, R.G., Monroy, F. Phys. Rev. Lett., 2004, 92,255503

[67] Donth, E.J., Relaxation and thermodynamics in polymers; Akademie Verlag: Berlin, 1992.

[68] Sacchetti, M., Yu, H., Zografi, G. Rev. Sci. Instrum. 1993, 64 , 1941.

[69] Hilles, H., Maestro, A., Monroy, F., Ortega, F., Rubio, R.G., Velarde, M.G. J. Chem. Phys. 2007, 126, 124904.

[70] Keddie, J.L., Jones, R.A.L., Cory, R.A. Europhys. Lett., 1994, 27, 59.

[71] Forrest, J.A., Mattson, J. Phys. Rev. E 2000, 61, R53.

[72] Sanchis, A., Prolongo, M.G., Masegosa, R.M., Rubio, R.G. Macromolecules, 1995, 28, 2693.

[73] Ngai, K.L. Eur. Phys. J. E 2002, 8, 225.

[74] van Megen, W., Underwood, S.M. Nature, 1993, 362, 616

[75] Casalini, R., Roland, C.M. Macromolecules, 2005, 38, 1779.

[76] Cicutta, P., Hopkinson, I. J. Chem. Phys., 2001, 114, 8659.

[77] Cook, J.T.E., Richards, R.W. Eur. Phys. J. E 2002, 8, 111. 